\title{
LES EVOLUTIONS APPORTEES PAR LA REFORME DES FINANCES PUBLIQUES DANS LES SECTEURS DE LA SANTE ET L'EDUCATION
}

\author{
Par NDJEKO KALUME L'AEA ALEXIS et MWAPE NGOSA*
}

INTRODUCTION

La République démocratique du Congo se retrouve toujours dans la zone rouge en matière de gestion des finances publiques, elle affecte moins de ressources aux secteurs prioritaires que sont la santé et l'éducation. Le pays est aussi caractérisé par la mauvaise gouvernance. "Ce qui explique de graves dépassements budgétaires » Les efforts entrepris dans la réforme de ces secteurs n'ont pas encore produit des avancées significatives, ${ }^{1}$

*Situation politico administrative

Qualifié de scandale géologique, le sous-sol de la RD du Congo regorge de plusieurs minerais et d'énormes réserves énergétiques. En dépit de toutes ces immenses ressources humaines et de sous-sol, la RDC est classé parmi les pays les plus pauvres du monde. Certains indicateurs l'alignent parmi les pays les plus misérables de l'Afrique au sud du Sahara. Près de $80 \%$ de sa population survivent à la limite de la dignité humaine, avec moins de US \$ 0.20 par personne et par jour.

A ce qui précède s'ajoute le manque de volonté politique,le pays (RDC) se trouve confrontée à d'immenses défis pour fournir des services dans les domaines: DE L' EDUCATION ET DE LA SANTE

Les articles 42, 43, 47 de la constitution sont claire mais il se pose un problème de l'applicabilité concernant ces secteurs :

Art 42: Les pouvoirs publics ont l'obligation de protéger la jeunesse contre toute atteinte à sa santé, à son éducation et à son développement intégral.

Article 43: Toute personne a droit à l'éducation scolaire. Il y est pourvu par l'enseignement national

L'enseignement national comprend les établissements publics et les établissements privés agréés.

La loi fixe les conditions de création et de fonctionnement de ces établissements.

Les parents ont le droit de choisir le mode d'éducation à donner à leurs enfants.

L'enseignement primaire est obligatoire et gratuit dans les établissements publics.

* Assistants à l'Université de LUBUMBASHI.

1 Sissoko Fili, spécialiste en gestion financière à la Banque mondiale, lors d'un atelier tenu jeudi 29 mars 2012 à Kinshasa par le comité d'orientation de réforme des finances. 
Article 47: Le droit à la santé et à la sécurité alimentaire est garanti. La loi fixe les principes fondamentaux et les règles d'organisation de la santé publique et de la sécurité alimentaire(1)

La République Démocratique du Congo a souscrit, sur le plan international, à la déclaration universelle des droits de l'homme en son article 25 Stipule : «Toute personne a droit à un niveau de vie suffisant pour assurer sa santé, son bien-être et ceux de sa famille, notamment pour l'alimentation, l'habillement, le logement, les soins médicaux ainsi que pour les services sociaux nécessaires; elle a droit à la sécurité, en cas de chômage, de maladie, d'invalidité, de veuvage, de vieillesse ou dans les autres cas de perte de ses moyens de subsistance par la suite de circonstances indépendantes de sa volonté... » a l'adhésion à l'organisation mondiale de la santé «les soins essentiels fondés sur des méthodes et des techniques pratiques, scientifiquement valables et socialement acceptables, rendus universellement accessibles à tous les individus et à toutes les familles de la communauté avec pleine participation et à un coût que la Communauté et les pays puissent assumer... » 4 à l'objectif social de la santé pour tous «Santé pour tous d'ici l'an 2000... » Aux résolutions de la conférence internationales sur les soins de santé primaire d'Alma Ata; à la charte africaine des droits de l'homme et des peuples qui à son article 16 stipule que: « Toute personne a droit de jouir du meilleur état de santé physique et mental. »

Les Etats parties à la présente Charte s'engagent à prendre les mesures ${ }^{2}$ nécessaires en vue de protéger la santé de leurs populations et de de leur assurer l'assistance médicale en cas de maladie. »

Ainsi qu'à la déclaration des chefs d'Etat et de Gouvernement de l'organisation de l'unité africaine sur la santé comme base de développement.

La politique mondiale de la santé est basée sur le principe de «santé pour tous et tous pour la santé ».et cela avec une médecine scientifiquement prouvée et à la portée de tous

Sur le plan national, plusieurs textes réglementaires existent et comprennent divers ordonnances-lois relatives à la santé et a l'éducation. Cependant à ce jour, le système sanitaire et éducationnel congolais est marqué par une inadéquation entre la volonté politique éclairé et l'état des lieux réel.

Parmi les divers facteurs à la base de cette inadéquation, il y a lieu de relever ceux liés aux textes réglementaires eux-mêmes, en l'occurrence de l'absence de certains aspects pourtant indispensable, tels que les normes et réglementations nouvelles et/ou contradictoires de surcroit dilues dans plusieurs textes épars.et d'autre part le manque de volonté politique

$\mathrm{Vu}$ ces facteurs, ces secteurs présentent des faiblesses graves, c'est pourquoi cette étude va chercher à analyser : les Evolutions apportées par la réforme des finances publiques dans les secteurs de la sante et l'éducation. Car la dites reforme a une des grandes missions d appuyait fortement ses secteurs.

2 PNUD, Rapport sur les Objectifs du Millénaire pour le Développement. RDC, Kinshasa mars 2010. 
LA question est celle de savoir : est ce que la situation a telle évoluée vers les seuils universellement acceptable? La réponse est apparemment positive grâce aux concours des ménages, les privés (confessions religieuses, les O.N.G. et les entreprise para étatique) et financement extérieur. S'il faut compter sur le budget de l'ETAT $<6 \%$ pour un pays sans route, manque d'infrastructure et en poste conflit la situation allait être catastrophique.

C'est ainsi que dans un premier, nous allons analyser l'état sanitaire et dans un second temps nous analyserons l'état éducationnel en RDC.

\section{ETAT SANITAIRE DE LA RDC}

La santé est un état de complet bien-être physique, mental et social, et ne consiste pas seulement en une absence de maladie ou d'infirmité 3

Chaque personne a droit a la sante cela suppose que les pouvoirs publics doivent des conditions telles que chacun puisse jouir du meilleur état de santé possible. Ceci implique notamment l'existence de services de santé, des conditions de travail sûres et saines, un logement adéquat et une alimentation nutritive

La RDC, émergent d'une longue période de conflits, sa situation sanitaire, le manque de données ne permet pas d'obtenir une vision correcte sur les progrès éventuels des impacts durant ces dernières années au niveau national

Néanmoins, le diagnostic de la situation sanitaire du pays relève de nombreux défis; le niveau de santé des populations même si certains indicateurs se sont améliorer reste insuffisant en terme de morbi - mortalité : infantile, maternelle $1,{ }^{4}$ VIH/SIDA, PALUDISME, la TUBERCULOSE, TRIPANOSOMIASE (La maladie du sommeil reste un combat de tous les instants La RD Congo détient le triste record de la maladie du sommeil dans le monde. $)^{5}$

Devant cette situation, beaucoup ont disserté sur la situation de la santé en publique en RDC. A ce sujet, nombre de réflexion démontre l'état comateux dans lequel git le système sanitaire. Cette situation, à lire les écrits des gens qui voudraient la voir s'améliorer, ne date pas d'aujourd'hui, elle daterait de la deuxième république. Pendant cette période, le système sanitaire a vu son outil de travail détruit et non renouvelé; l'on a assisté pendant la même période précitée au non entretien sociale du personnel soignant (salaire dérisoire), à la fuite des cerveaux qualifiés vers d'autres cieux, porteurs d'espoir. L'état n'a pas créé des centres hospitaliers, ceux qui existaient sont soit non revitalisé soit détruits...

A la lecture de ce qui précède, on est en droit d'affirmer que les gouvernements d'après la deuxième république ont hérité d'un système sanitaire malade. Mais qu'ont-ils fait à leur tour pour sortir le sanitaire de son coma? Dans le cadre de la formation du personnels soignants (médecin, assistants médicaux, sage femme, nutritionnistes etc....), l'Etat congolais organise des facultés de médecine a diverses filières; la société peut-elle à l'heure actuelle

3 La définition de la santé de l'OMS.

4 DOCUMENT INTERIMAIRE DE STRATEGIES DE REDUCTION DE LA PAUVRETE.

5 Mar-21 Jui Écrit par Syfia Grands Lacs. 
se féliciter des service qu'elle reçoit des filles et des fils que l'université met à sa disposition pour résoudre le problèmes de la santé? L'analyse de notre thème devra nous permettre de signifier, si état serait en droit de prétendre dire qu'il y aurait de progrès dans le domaine. Certes, l'Etat congolais organise l'enseignement supérieur et universitaire et recherche scientifique. Le souci majeur est de former des cadre susceptible de répondre aux problèmes qui frappent la nation; la faim, la santé en générale, développement, etc. la RDC, compte des universités, des instituts supérieurs, des écoles facultaire d'où sortent des hommes de recherche. Mais ces hommes qui en sortent, de quel niveau intellectuel et professionnel sont-ils, si l'on sait dans quelles conditions ils étudient? Parlant de la faculté de médecine, ce sont des médecins non connectés à la technologie moderne de la médecine; et même s'ils l'étaient c'est un climat social malsain ou ils évoluent : honoraire insignifiants, salaire dérisoire.... Sans revenir sur la formation du personnel professionnel de santé parce que la question sera débattu dans le point de l'éducation, mais de quelle manière sont administrés les soins dans la plupart des maisons des soins (hôpitaux, polyclinique, dispensaires).

Dans le cas concret sur le plan pratique (stage pendant la formation) dans la ville les étudiants en médecine parfois n'ont pas des maitres encadreurs sur terrain, ils sont encadrés soit par les anciens infirmiers(es) ou les anciens médecins généralistes qui ne consacrent pas leurs temps pour leurs encadrements, même si ils ont les maitres de stage ils ne passent que pour certaines formalités. En matières d'encadrement ils sont autodidactes pour leur propre formation. Tout comme dans d'autre formation hospitalière il $\mathrm{n}$ ya que seulement les étudiants stagiaires qui assurent les soins et les interventions médicales, ils s'en sortent tant bien que mal.

Un des plus grands éléments a souligné ou un grand service salvateur de l'université de Lubumbashi via la Faculté de médecine est le grand service de la gestion de l'hôpital général de référence HOPITAL SENDWE (étatique) de la province, un hôpital a la bourse presque de la population locale (même les 3 provinces voisines : le MANIENA et les 2 KASAI)et qu'on retrouve beaucoup d'indigents, la faculté remplie pleinement sont rôle de recherche, de soigner...malgré le maigre moyen sans le concours presque de l'Etat, la faculté donne les personnels qualifiés, l'Etat cède le bâtiment et la population se prennent en charge.

C'est au cout d'argent que tout se fait; c'est normal.Mais au-delà de cette question d'argent, il y a l'aspect humanitaire et, c'est cet aspect humanitaire qui devrait plus préoccuper l'Etat, c'est-à-dire les gouvernements. Cette question reste au niveau de bonne intention. Dans les milieux sanitaires, les malades reçoivent les soins après avoir versé l'argent. Le contraire n'est pas accepté. La conséquence les malades deviennent leurs marchandises. Si l'enseignant congolais a résolu le calvaire par les F.I.P (fond d'intervention ponctuel), les professionnels sont loin de sauver leur peau, celui-ci qu'il reçoit est déjà essoufflé, en argent et en force. Comme on peut le constater l'image de la santé au Congo démocratique est à plaindre. Face à cette réalité, quelles sont les prouesses de l'état pour améliorer cet état des choses? L'on peut constater des dons en médicaments dans diverse centre de santé, hô- 
pitaux. Les partenaires extérieurs, les organisations internationales viennent en aide pour épauler les gouvernements. De même, les dons de cette coopération ne sont pas directement des bénéficiaires aux destinataires, plusieurs fois on entend que tel stock des médicaments, don ou lege de tel organisme a été détourné par les professionnels des santés ou par un haut dignitaire, cela a été détourné(sans aucune forme de procès) L'immoralité a remplacé la moralité. Cette réalité est vivante, aujourd'hui on ne s'étonne pas de voir un haut fonctionnaire, un homme d' affaire ou ancien professionnel de santé de l'état devenir propriétaire d'un centre de santé avec la quasi totalité du médicament ou des matériels des soins, vu cout élevé part rapport a la bourse des ménages la population diminue recours à la médecine de la rue, avec comme conséquence le taux élevé de morbi-mortalité. Devant cette situation le pays assiste a une grande prolifération de la médecine dites tradimoderne provenant de la Zambie, Kenya, Tanzanie, Nigeria, Australie, Inde et Chine évoluant dans un champ sans maitre...

\section{LE FINANCEMENT DE LA SANTE}

La République fait siens les objectifs du millenium pour le développement(OMD) qui devraient consister à:

- Réduire de 3/4 la mortalité maternelle d'ici 2015;

- Réduire de 2/3 la mortalité des enfants de moi de 5ans d'ici 2015;

- Stopper et inverser les tendances de la propagation du VIH/SIDA d'ici 2015.

Mais fâcheusement la RDC a commence les OMD avec un retard de 6 ans (2006) ...et pour y parvenir le concours de l'extérieur et para étatique sont incontournable.

Le système de financement du secteur de la santé en RDC repose principalement sur un trépied constitué du budget de l'Etat, des apports extérieurs (bilatéraux et multilatéraux) et du recouvrement des coûts des soins et services de santé auprès des usagers, a ce la s' ajoute les entreprises. De nombreuses études montrent que le financement extérieur porte essentiellement sur les investissements (immobiliers, équipements et médicaments). Par contre le fonctionnement des services est assuré majoritairement par les usagers et très peu par l'Etat et essentiellement sous forme de versements pour des salaires et des primes.Selon les comptes nationaux de la santé 2009 , les dépenses du secteur santé se répartissent comme illustré à la Figure 1: Répartition de dépenses nationales en santé par source de financement en \% en 2009

\begin{tabular}{|c|c|c|c|}
\hline Gouvernement central & Ménages & Fonds Extérieurs & Entreprises \\
\hline $12 \%$ & $42 \%$ & $36 \%$ & $10 \%$ \\
\hline
\end{tabular}

Le pays compte actuellement 493 hôpitaux dont 261 appartement l'Etat 165 aux confession religieuses 65 aux entreprises des secteurs public et privé;7725 autres établissements de soins comprenant les centre de santé de référence, les centres de santé, les maternités, les dispensaires et les polycliniques appartement également à l'Etat, aux entreprises, aux 
confession religieuses, aux ONG et aux personnes privées physiques et morales. ${ }^{6}$ Le plus grand nombre des hôpitaux Etatique sont dans un état de délabrement, manque des matériels, carences en médicaments (hôpitaux non revitalisé) si il ya un réunissant encore les critères de viabilité et de fiabilité cela est le fruit soit d'une ONG et/ou d'un organisme international ou encore un don...

\section{LE BUDGET DE L'ETAT CONSACRE A LA SANTE}

La part du budget de l'état consacré à la santé reste toujours très inférieure aux engagements des chefs d'Etat à ABUJA en 2001 (soit 15\%). De fait, il est resté intérieur à $2 \%$ du budget global de l'Etat entre 1998 et 2000, il est remonté à $3 \%$ avant de retomber à moins de $1 \%$ en 2000 .

\begin{tabular}{|l|c|c|c|c|c|c|}
\hline FINANCE & $\mathbf{2 0 0 5}$ & $\mathbf{2 0 0 6}$ & $\mathbf{2 0 0 7}$ & $\mathbf{2 0 0 8}$ & $\mathbf{2 0 0 9}$ & $\mathbf{2 0 1 0}$ \\
\hline $\begin{array}{l}\text { Total de dépense de santé en } \\
\text { \% du PIB }\end{array}$ & $3,4 \%$ & 2.9 & 3.5 & 7.4 & 7.9 & 2.8 \\
\hline $\begin{array}{l}\text { Dépense publique de santé } \\
\text { en\% du total de dépense de } \\
\text { santé }\end{array}$ & 4.6 & 4 & 6 & 15 & 12 & 3 \\
\hline $\begin{array}{l}\text { Ressources externes pour la } \\
\text { santé en \% }\end{array}$ & 15 & 14 & 14 & 34 & 36 & 8 \\
\hline $\begin{array}{l}\text { Total de dépense de santé } \\
\text { par habitant (US \$) }\end{array}$ & 3.9 & 4.1 & 5.3 & 13 & 13 & 4.7 \\
\hline $\begin{array}{l}\text { Dépense publique de santé } \\
\text { par habitant (US\$) }\end{array}$ & $<1$ & $<1$ & 2 & 2 & 2 & 1 \\
\hline
\end{tabular}

Ensuite il a augmenté jusqu'à culminer en 2004 avec 7\%.

Pour les années suivantes (de 2005 à 2010) les indicateurs sont présentés dans le Tableau 6 : Principaux indicateurs sur le financement de la santé en RDC.

Malgré ses engagements, le gouvernement Congolais ne parvient donc pas à consacrer davantage de moyens financiers à la santé. ${ }^{7}$

Un autre problème important est celui du taux de décaissement. En effet, si ce dernier a également évolué avec le temps depuis 2001, il est resté en moyenne de 54\% sur la période allant de 1998 à 2009 si l'on ne prend pas en compte des pics liés à des dépenses d'urgence ou à des augmentations des primes et salaires intervenues en plein exercice budgétaire (6).

Une seconde caractéristique concerne l'insuffisance de priorisation dans l'affectation des ressources, voire des incohérences entre les priorités déclarées et les financements alloués.

6 PNLP:“ faire reculer le paludisme "plan stategique 2009-2013.

7 Ces données fournies par les Comptes Nationaux de la Santé le 20 juillet 2011. 


\section{LES PARTENAIRES EXTERIEURS}

Sur les dix dernières années, la République démocratique du Congo (RDC) a progressé en matière de survie de l'enfant et d'éducation, indiqué le Fonds des Nations Unies pour l'enfance (UNICEF) lors de la publication de sa dernière enquête à indicateurs multiples sur le pays.

"Cette enquête démontre que nous avons fait des progrès significatifs durant les dix dernières années $»,{ }^{8}$

Pour parachever cette partie, disons que l' état sanitaire de la République s 'est amélioré grâce aux concours des plusieurs mains, nous citons l'Unicef, l'Unesco, la Banque mondiale, L'USAID et les coopérations française, espagnole et belge font partie des partenaires financiers et techniques de la RDC dans le secteur de l'éducation et de la santé, sans pour autant oublier les confessions religieuses, les ONG locales et internationales, les ménages et les entreprises para étatiques.

\section{EDUCATION}

Les évolutions apportée dans le secteur de L'éducation travers la reforme de finance publique n'ont pas encore produit des avancées significatives, cela reste dans les rubriques des bonnes intentions

L'éducation est le capital d'investissement de chaque nation, tous les gouvernements qui se succèdent en République démocratique du Congo sont conscients de cette évidence.

C'est ainsi qu'on lira dans certains rapports sur ce secteurs des réflexions telles que :

1. l'éducation est la base de tout développement et demeure la condition fondamentale du succès de la stratégie de développement du gouvernement.

2. l'éducation constitue une condition indispensable, sinon suffisante du développement de l'individu et de la société, elle contribue à améliore la sécurité, la santé et favorise le progrès social, économique et culturel, surtout dans un système de lutte contre la pauvreté.

3. «En rapport avec l'éducation,189 nations et 147 chefs d'état réunis en 2001 a ABOUJA s'étaient fixé des objectifs du millénaire pour le développement à atteindre en 2015, ${ }^{9}$ A la lecture de ces trois considérations, nous constatons que le développement de l'individu et de la société passe nécessairement par l'éducation.

D'où le souci qu'ont les Etas moderne (les gouvernements) à améliorerez leur système éducatifs

Eu égard ce qui précède, une problématique mérite d'être analysée par rapport au système éducatif c'est-à-dire, le système éducatif de la République démocratique du Congo

8 La Représentante de l'UNICEF en RDC, Pierrette Vu Thi, lors du lancement du document «congo PLANETE » à Kinshasa, la capitale congolaise.

9 - Revue de la situation des OMD en RDC (Septembre 2010.). 
Le gouvernement congolais investit-ils les moyens nécessaires pour l'amélioration du système éducatif?

Les quelques indicateurs avancés par le gouvernement permettent -ils de considère une quelconque amélioration?

N'est ce pas l'apport des parents congolais et des partenaires internationaux (l'Unicef, l'Unesco, la Banque mondiale, L'USAID et les coopérations française, espagnole et belge, les religieux, les prives et les ménages, mériterait plus d'être salué dans la survie de l'éducation congolaise?

L'analyse de ces trois questions nous permettrons de peindre à notre manière l'image de système éducatif.

L'éducation est le levain du développement intégral d'un individu, d'une nation, d'un pays etc....

Elle s'intéresse à tous les aspects de la vie : social, économique; politique, religieux etc. les institutions du pays ne sont pas sans ignorer cet aspect de la question, en référence des articles 42, 43 «Toute personne a droit à l'éducation scolaire. Il y est pourvu par l'enseignement national.

L'enseignement national comprend les établissements publics et les établissements privés agréés.

La loi fixe les conditions de création et de fonctionnement de ces établissements. Les parents ont le droit de choisir le mode d'éducation à donner à leurs enfants.

L'enseignement primaire est obligatoire et gratuit dans les établissements publics.»,47, de la constitution de la RDC. ${ }^{10}$

L'enseignement national exige des moyens conséquents de la part de qui organise, le gouvernement.

\section{FINANCEMENT}

\section{a) L'Etat Congolais}

Le secteur éducatif congolais, le gouvernement loue un budget très insignifiant qui ne favorise pas la rémunération de l'agent formateur, «l'enseignant». Il n'est pas étrange en République Démocratique du Congo de rémunérer l'enseignant par 20 000-50 000 franc congolais $(22$ a $55 \$)$ par mois.

Dans les années 60, après l'accession du pays à l'indépendance, l'Etat congolais réservait au moins $25 \%$ de son budget à l'éducation nationale. Ces moyens conséquents, couples avec un partenariat efficace dont l'école publique bénéficiait de la part des confessions religieuses, ont fait du secteur de l'enseignement l'un des plus importants sur lesquels pouvait compter le gouvernement pour un meilleur processus du développement national. L'enseignement était alors un noble métier. Mais, a ces jours, le secteur de l'enseignement fait fi- 
gure de parent pauvre et affiche un tableau le plus sombre en RDC, avec une enveloppe budgétaire qui nage pratiquement dans les $6 \%$.qui crée le Scepticisme dans les écoles « le Directeur adjoint de l'école primaire I de la commune de Barumbu; Liévin Muabilua relève la modicité du pourcentage du budget alloué à l'éducation et s'insurge contre son emploi. «Réellement, 2\% seulement sont utilisés pour le paiement et les 4 autres disparaissent dans la nature », dénonce-t-il.

Les professionnels de ce secteur, essentiellement les enseignants, demeurent sceptiques, de la part réservée à l'éducation dans le budget de l'Etat exercice 2011. La prévision s'élève à plus de 600 millions USD. L'éducation étant un secteur retenu dans le programme des 5 chantiers du gouvernement, la question est de savoir si ce montant suffit pour faire fonctionner plus de 26.000 écoles en RDC.

Près de $70 \%$ des établissements d'enseignement public sont gérés par des confessions religieuses (écoles conventionnées) alors que moins de $20 \%$ le sont directement par l'État (écoles non conventionnées, dites « officielles »).

Le reste est constitué d'écoles privées (10\%). Les écoles privées sont particulièrement nombreuses en zones urbaines.

La qualité du système éducatif est également un sujet de préoccupation. S'agissant de formation des enseignants, le personnel enseignant souffre d'une formation initiale jugée peu professionnalisante et de l'absence quasitotale de système national fonctionnel de formation continue. Selon les données statistiques 2007/2008, un tiers des enseignants du primaire et $64 \%$ des enseignants du secondaire n'ont pas la qualification requise pour enseigner.

Le cumul de ces deux facteurs a provoqué une rupture professionnelle et intellectuelle du corps professoral menant à la détérioration de la qualité de l'éducation. Les budget de l' enseignement a la hausse 5.607,5 milliards l'exercice budgétaire 2010 a 562,2 milliards de franc congolais (FC)l'exercice budgétaire 2011 un taux d'accroissement nominal de $14,9 \%$. N'avoisine pas les recommandations de la CMD «L'augmentation du pourcentage du budget affecté à l'éducation est encore bien loin d'atteindre les $20 \%$ d'affectation au secteur de l'éducation (uniquement jusqu'au niveau du premier cycle de l'enseignement secondaire) préconisés par la Campagne mondiale pour l'éducation». ${ }^{11}$ Pour premier ministre MUZITO, le 14,9\% cela va aussi assurer la gratuité de l'enseignement de base (projet non réalisé, en violation meme de la constitution en son art 43 «....L'enseignement primaire est obligatoire et gratuit dans les établissements publics.») et compenser la prise en charge des parents dans le fonctionnement des écoles et des bureaux de gestion, ce crédit servira à la mécanisation de 17.000 enseignants (16 milliards Fc), au fonctionnement de plus de 26.000 écoles à travers le pays ( 8 milliards Fe trimestriellement), au fonctionnement des bureaux

11 Par Professeur Gratien Mokonzi; Faculté de psychologie et des sciences de l'éducation Université de Kisangani. 
de gestion (11 milliards Fc) à l'acquisition des objets scolaires milliards Fc) et à la réhabilitation et reconstruction des écoles (34 milliards Fc). ${ }^{12}$

Le gouvernement considère le montant de 562,2 milliards $\mathrm{Fc}$ affectés à l'éducation comme des efforts de l'Etat en vue d'améliorer l'enseignement en RDC. « Ce montant sera destiné à la construction et la réhabilitation des écoles, à l'amélioration des conditions scolaires », A.MUZITO. ${ }^{13}$ Mais, ce qui est peut-être encore plus grave ce crédit alloué, il n'y a pas de système de gestion transparent et efficace des fonds alloués actuellement.

Il n'existe aucune publication indiquant la ventilation par poste du budget de l'éducation. Les documents et rapports budgétaires détaillés sur les dépenses ne sont accessibles à personne en dehors du ministère, ce qui rend impossible de connaître les sources et les objets du financement ou d'évaluer dans quelle mesure les dépenses suivent réellement les priorités fixées dans le processus de planification. C'est dans cette réalité qu'œuvre l'éducateur congolais comme on peut l'imaginer, c'est avec un moral entamé donc sans conscience que l'enseignant prétend façonner les futurs cadres de demain.

François Rabelais, auteur français du 16 ${ }^{\mathrm{e}}$ siècle écrivait : " science sans conscience n'est que ruine de l'âme » c'est vrai, l'âme congolaise est ruinée »

En 2002, une étude menée par la banque mondiale dénommée »le renouveau du système éducatif de la république démocratique du Congo »(RESEN) a identifié quatre problème important qui minent l'accès et qualité de l'enseignement en RDC à savoir :

1. Une couverture relativement faible au niveau primaire avec des inégalités dans l'accès et une extension incontrôlée au niveau du secondaire et du supérieur.

2. Une grave détérioration de la qualité de l'éducation à, tous les niveaux

3. Un système administratif scolaire

4. Un Très bas niveau de dépense et un système de financement inefficace et inéquitable

A lire ces quatre constats des organismes internationaux, nous osons dire que les articles 42 et 43 de la constitution restent des simples intentions mais inapplicable en réalité.

Si l'on peut encore parler de l'éducation en RDC le grand apport est à mettre à, l'actif de l'extérieur

\section{b) Les parents d'élèves}

Hormis le budget (3\%) de l'État, ce sont les parents d'élèves qui sont devenus les patrons de l'enseignement national du Congo démocratique. Leur contribution ne se limite pas à, payer les frais leur reconnu mais elle va même dans l'enveloppe salariale du personnel enseignant : c'est le phénomène FIP (Frais d'Intervention Ponctuelle) a l'origine en 1990, les FIP devraient suppléer à l'enveloppe salariale dérisoire.

... Mr Kabeya parent de 5 enfants : 2 a l'école secondaire, un a l'institut NYOTA et 1 'autre au Lycée TUENDELEE, obligé de payer 33000 francs congolais $( \pm 36 \$)$ et autre au 
lycée TUENDELEE 60.000 FC (en raison de $20000 \mathrm{fc}$ par enfant et par moi $( \pm 62 \$)$ et l'ensemble fait la somme $93000 \mathrm{FC}( \pm 1 \mathrm{O} 2 \$ /$ mois $)$ pour un fonctionnaire de $l^{\prime}$ Etat qui touche $37.500 \mathrm{FC}( \pm 41 \$)$ par mois. A côté de cela le loyer mensuel, le payement de l'eau et d'électricité, la ration alimentaire et autre, cela est l'une des causes de la dégradation de niveau d 'enseignement par ce que vu cette inadéquation entre le salaire et les frais d'intervention ponctuel a payer beaucoup de parents ne veulent pas que leurs enfants reprennent la classe, ils font tout pour négocier les points chez l'enseignant (lui aussi dans la même situation) et devant cette situation comme la décentralisation n'est encore en application, l'Etat au niveau central est responsable.

Pour l'initiateur l'église catholique, ces FIP ne devraient pas durer long feu car, les pour- parler dans le cadre de l'amélioration du salaire étaient en cours (d'après le ministre de l'éducation nationale]; mais hélas, les FIP demeurent jusqu'à aujourd'hui entrainant derrière eux plusieurs conséquences sociales tant du côté du formé que du côté du formateur

L'enseignant est chosifié, car sa survie est dans les poches des parents c'est constat de la mémoire collective.

D'autres décisions du gouvernement restent au niveau des intentions quelle que soient les prétendues avancées relevé par le dit gouvernement Bien qu'universellement reconnu comme inaliénable, le droit à l'éducation n'est malheureusement pas encore assuré à tous, et surtout aux citoyens des couches sociales démunies en République Démocratique du Congo (R.D.C). certes les pays traverse une crise socio-politique et économique qui a affaibli l'Etat, le rendant incapable de remplir ses responsabilités dans divers domaines dont celui de l'éducation qui nous intéresse ici.

Ces dernières années, le secteur de l'éducation du pays est caractérisé par l'exigence de prise en charge financière de l'éducation à tous les niveaux par les parents des élèves et étudiants. Cette contribution est devenue obligatoire et consacrée par le concept de partenariat1(*) entre parents d'élèves et les professionnels de l'éducation ou les établissements scolaires et universitaires d'enseignement. ${ }^{14}$

Cependant, les difficultés liées à la modicité des salaires des parents (pour ceux qui travaillent), à l'irrégularité de payement de ces salaires, au chômage et à l'insuffisance des revenus ménagers, ont placé beaucoup de parents dans l'incapacité de prendre en charge la scolarité de leurs enfants. Ce, à cause du coût relativement élevé des études dans un tel contexte de la société

\section{c) Les Partenaires}

La Banque Mondiale est une institution active dans le secteur. Elle finance notamment le Projet d'Appui au Redressement du Secteur de l'Education (PARSE) depuis 2008 pour une

14 Le Partenariat est devenu un mode de gestion surtout financière des écoles et universités en R.D.C. Dans ce mode, les parents sont obligés de suppléer aux salaires insuffisants des enseignants et au fonctionnement des établissements scolaires et universitaires. Il est devenu une condition sine qua none de l'ouverture des années scolaires et académiques!). 
durée de cinq ans. Le projet se fixe essentiellement comme objectif de renforcer les capacités institutionnelles et financières du secteur de l'éducation en préparant le terrain pour le développement et le financement durables du secteur en vue de faciliter la coordination des donateurs et la transition future vers un programme couvrant l'ensemble du secteur de l'éducation. On compte notamment au titre des activités du PARSE au niveau de l'enseignement primaire : la construction scolaire, la distribution des manuels scolaires, la mécanisation (salarisation) d'enseignements supplémentaires ainsi que l'appui au développement des stratégies des sous-secteurs éducatifs (trois ministères en charge de l'éducation).Le projet PURUS de la Banque Mondiale, quant à lui, assure le financement des frais de fonctionnement des écoles secondaires.

L'UNICEF a concentré ses activités sur la petite enfance à travers la construction de plus de cinq cent centres de développement de la petite enfance dans lesquels plus de mille cinq cents éducateurs ont suivi des formations. Par ailleurs, il œuvre pour l'amélioration de la qualité de l'enseignement primaire en distribuant des kits didactiques et scolaires à plus de trois millions d'élèves et soixante-dix sept mille enseignants. A cela s'ajoute des formations dispensées à plus de treize mille enseignants et la réhabilitation de cinquante et une écoles.

D'autres partenaires tel qu'USAID concentre leur stratégie sur l'amélioration de l'accès à une éducation de qualité et l'accroissement de l'accès à l'éducation des populations vulnérables. Trois projets sont au cœur de cette stratégie de coopération : le programme de l'ambassadeur pour la scolarisation des filles qui promet l'accès et le maintien, le Projet de stabilisation de l'éducation de base à l'est de la RDC qui a notamment formé près de sept mille enseignants et fournit du matériel scolaire et didactique et le programme d'amélioration de la qualité de l'éducation qui doit former près de vingt mille enseignants et éducateurs, réhabiliter douze mille classes et fournir plus de neuf cent mille kits à des écoles et des enseignants dans trois provinces de la RDC. L'axe programmatique de la coopération américaine se concentre sur la formation continue des enseignants du primaire, avec un projet d'environ 22 millions de dollars pour les cinq prochaines années

La Belgique est également un partenaire actif dans le secteur éducatif à travers la Wallonie Bruxelles Internationale, la Coopération Technique Belge (CTB), l'Association flamande pour la coopération au développement et l'assistance technique (VVOB), la Vlaamse Interuniversitaire Raad (VLIR). La Belgique apporte un appui au développement des compétences en matière de mise en œuvre des nouvelles technologies de la communication et de gestion des réseaux informatiques au Centre de Documentation de l'Enseignement

Supérieur et Universitaire et de la Recherche de Kinshasa. Dans le domaine de la formation professionnelle, l'appui prend la forme de la restructuration des centres de formation professionnelle par le biais d'une intervention auprès de trois centres de formation professionnelle pilotes de Kinshasa et auprès du ministère de la jeunesse et des sports

Ces résultats encourageant obtenu dans le secteur de l'éducation, âpres une évolution importante des indicateurs pendant un temps sont dus à l'appui de l'Unicef à travers la campagne «tous les enfants à l'école » qui a fortement contribué à l'augmentation des effectifs 
scolaires quelque soit le sexe, ainsi que pédagogique et la réhabilitation des infrastructures scolaires»

Comme toujours au chevet de la RDC, l'Unicef, l'Unesco, la Banque mondiale, l'Usaid et les coopérations française, espagnole et belge font partie des partenaires financiers et techniques de la RDC dans le secteur de l'éducation et de la santé, sans pour autant oublier les confessions religieuses, les ONG locales et internationales, les ménages et les entreprises para étatique. ${ }^{15}$

\section{CONCLUSION}

Les secteurs de L' EDUCATION et de la SANTE font figure d'un parent pauvre et affiche un tableau le plus sombre, avec une enveloppe budgétaire qui nage pratiquement dans le $6 \%$ pour une population estimée environs a plus 60 millions d'habitants dont le revenu annuel par personne est < à 125 \$, LA SITUATION du pays reste préoccupante en général et en particulier dans les zones rurales. L'Etat affecte moins de ressources aux secteurs prioritaires, faisant allusion à l'étude sur l'évaluation de la marche de la République démocratique du Congo (RDC) vers la réalisation des Objectifs du Millénaire pour le Développement (OMD) menée en prévision du Sommet sur l'évaluation des OMD de septembre 2010, le pays ne pourra pas réaliser tous les OMD à l'horizon 2015. Globalement, les progrès réalisés de 2000 à 2010, sont très timides. Il y a cependant lieu de noter que la stratégie congolaise de promotion de la croissance et de réduction de la pauvreté a été mise en place en 2006, soit six années après la signature du Pacte du Millénaire. Si l'on s'en tient à cet état de choses, on devrait logiquement repousser l'horizon temporel à 2021.

Le tableau actuel que présente les pays :les avancées significatives avec les indices objectivement observables sont les fruits d 'un bon partenariat avec l'Unicef, l'Unesco, la Banque mondiale, l'Usaid et les coopérations française, espagnole et belge, sans pour autant oublier les confessions religieuses, les ONG locales et internationales, les ménages et les entreprises para étatique. Les efforts entrepris dans la réforme de ces secteurs n'ont pas encore produit des avancées significatives, cela Est classé dans les initiatives des bonnes intentions. Il faut que le gouvernement s'implique pour améliorer la situation socio-économique du pays car la grande majorité des populations congolaises est pauvre et cette pauvreté présente plusieurs facettes dont : la faiblesse du revenu, la non satisfaction des besoins alimentaires, l'incapacité d'accéder aux soins de santé et à la scolarisation, l'incapacité de se loger décemment, cela est 1 une des raisons qui fond classer le pays au rang des PAYS LES MOINS AVANCES (PNUD)se retrouve toujours dans la zone rouge en matière de gestion des finances publiques, en affectant moins de ressources aux secteurs Education et SANTE,Ces efforts sont à déployer à différent niveaux de vie Nationale. Que budget, alors cadre juridique puisse dans sa reforme ultérieure (budget 2014,2015...) puissent accorder une part importante que sont la sante et l'éducation

15 Revue de la situation des OMD en RDC (Septembre 2010) PNUD. 


\section{BIBLIOGRAPHIE}

\section{TEXTES DES LOIS}

1. LOI $\mathrm{N}^{\circ} 1 \mathrm{O} / \mathrm{O} 13 \mathrm{du} 31$ DECEMBRE 2010 portant budget de l'Etat pour l'exercice $2 \mathrm{O} 11$

2. LOI $^{\circ} 11 / \mathrm{O} 11 \mathrm{du} 13$ JUILLET $2 \mathrm{O} 11$ relative aux Finances publiques.

\section{OUVRAGE}

BAKANDEJA WA MPUNGU Grégoire; Les Finances publiques : pour une meilleure gouvernance économique et financière en RDC; Larcier; Bruxelles;20O6

\section{DOCUMENTS DIVERS}

1. DOCUMENT UNESCO DE PROGRAMMATION PAYS REPUBLIQUE DEMOCRATIQUE DU CONGO 2011-2013 ADVANCE COPY/COPIE PRELIMINAIRE Mai 2011 Bureau UNESCO Kinshasa

2. Fourniture efficace de Services dans le domaine de l'enseignement publicPar Professeur Gratien Mokonzi Faculté de psychologie et des sciences de l'éducation Université de Kisangani Avec le concours de Madame Mwinda Kadongo inspectrice de l'enseignement primaire, secondaire et professionnel

3. DOCUMENT INTERIMAIRE DE STRATEGIES DE REDUCTION DE LA PAUVRETE, Kinshasa, mars 2002

4. Documentation des résultats de la mise en œuvre des principes de l'efficacité de l'aide dans le secteur de la santé

5. Véronique Zinnen, Etude de cas de la République Démocratique du Congo Version finale,février 2012 Université catholique de Louvain Faculté de santé publique, Bruxelles Veronique.Zinnen@uclouvain.be

6. Ministere du Plan: DOCUMENT DE STATEGIE POUR LA REDUCION DE LA PAUVRETTE (DRAFT Nº 01 POUR DISCUSSION),KINSHASA 2005

7. MUSA ALOKPO Dieudonné, EDUCATION NON FORMELLE DANS LES REPUBLIQUE DEMOCRATIQUE DU CONGO Etude exploratoire sur le statut légal et les attitudes des habitants de Kinshasa (D E A)

\section{SITES}

http://radiookapi.net/actualite/2012/03/30/gestion-des-finances-publiques-la-rdc-toujours-dans-la-zonerouge-selon-la-banque-mondiale/ 
LES EVOLUTIONS APPORTEES PAR LA REFORME DES FINANCES PUBLIQUES DANS LES SECTEURS DE LA SANTE ET L'EDUCATION

https://www.ammado.com/nonprofit/46502/articles/17057 (09/06/2012)

http://rdcmaps.centerblog.net/ (09/06/2012)

http://www.digitalcongo.net/article/82749 (23/07/2012)

http://rdcmaps.centerblog.net/rub-aspects-economiques-.html (01/08/2012) 